\title{
A Novel Antihepatitis Drug, Bicyclol, Prevents Liver Carcinogenesis in Diethylnitrosamine-Initiated and Phenobarbital-Promoted Mice Tumor Model
}

\author{
Hua Sun, Linghong Yu, Huailing Wei, and Gengtao Liu \\ Department of Pharmacology, Institute of Materia Medica, Peking Union Medical College \& Chinese Academy of Medical Sciences, \\ Beijing 100050, China \\ Correspondence should be addressed to Hua Sun, sunhua@imm.ac.cn
}

Received 27 July 2011; Revised 21 September 2011; Accepted 21 September 2011

Academic Editor: Masa-Aki Shibata

Copyright () 2012 Hua Sun et al. This is an open access article distributed under the Creative Commons Attribution License, which permits unrestricted use, distribution, and reproduction in any medium, provided the original work is properly cited.

Bicyclol, an antihepatitis drug developed by Chinese scientists, has been shown to prevent the malignant transformation induced by 3 -methylcholanthrene and 12-O-tetradecanoylphorbol-13-acetate in WB-F344 rat liver epithelial cells. This study provides further evidence on its role as a chemopreventive agent in experimental mice with diethylnitrosamine- (DEN-) initiated and phenobarbital- (PB-) promoted liver carcinoma. Liver tissue and serum were collected. In the two-stage model of hepatocarcinogenesis in mice, oral administration of bicyclol $(100,200 \mathrm{mg} / \mathrm{kg}$ ) before DEN injection showed significant reduction in the incidence of hepatocellular foci, nodules, or carcinoma. Histopathological examination revealed that there was no hepatocellular carcinoma (HCC) and hepatoma formation in the mice pretreated with bicyclol $(200 \mathrm{mg} / \mathrm{kg})$ at week 20 , while the mice treated with DEN/PB developed 33.3\% HCC and 55.6\% hepatoma. Furthermore, the serum levels of alanine aminotransferase (ALT), alkaline phosphatase (ALP), and $\alpha$-fetal protein (AFP) in serum significantly increased in the DEN/PB model group in comparison with the control group. Pretreatment with bicyclol showed a marked reduction in the above condition. Bicyclol also decreased the expression of AFP and proliferating cell nuclear antigen level in the liver tissue and attenuated the decrease in body weight. In this study, we also found that 10 weeks after stopping the administration of PB and drugs, the control and bicyclol-treated $(200 \mathrm{mg} / \mathrm{kg})$ animals showed no HCC and hepatoma formation at the time of termination whereas DEN/PBinduced mice developed 100\% hepatoma and 50\% HCC. These results further indicate that bicyclol has the chemopreventive potential for liver carcinogenesis induced by carcinogens.

\section{Introduction}

Hepatocellular carcinoma (HCC) comprises $90 \%$ of the liver tumors occurring worldwide in humans, with more than half a million new cases reported every year. Diagnosis of this cancer, once restricted mostly to Asia, is now rising in Europe as well as in North America [1]. Environmental factors, such as hepatitis virus infections and chemical carcinogen exposure, are causally implicated in HCC occurrence. About $80 \%$ of human HCC cases are attributable to infection [2]. Chronic hepatitis B virus (HBV) carriers are 100-400 times more likely to develop HCC than noncarriers [3]. Hepatitis $\mathrm{C}$ virus (HCV) is the second most common cause of HCC after HBV [4]. Currently, HCC represents more than $4 \%$ of all cancer cases in the world and causes at least 315,000 deaths every year [5]. Although early HCC can be cured by surgical resection, many HCC cases are asymptomatic; thus, most HCC patients are not diagnosed in time.

An effective approach to cancer control is chemoprevention, which is expected to interfere with the initiation, promotion, or progression of carcinogenesis. Generally, long-term therapy is required for both chronic HBV and $\mathrm{HCV}$. An antihepatitis drug can be considered to be of great clinical value if it inhibits or suppresses the development of hepatocarcinogenesis besides its improvement of abnormal liver function and inhibition of hepatitis virus replication. 


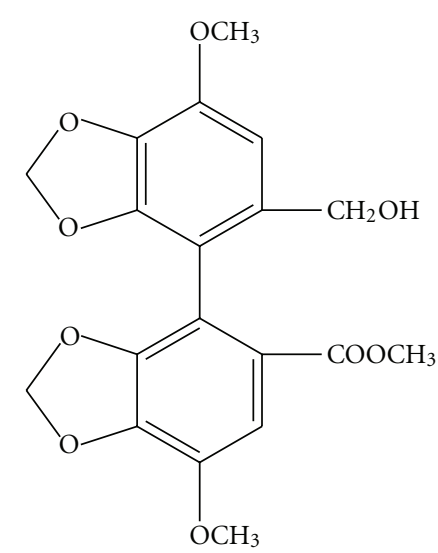

Figure 1: The chemical structure of bicyclol.

Bicyclol (4,4' -dimethoxy-2,3,2' $3^{\prime}$-dimethylene-dioxy-6hydroxymethyl-6' -carbonyl-biphenyl) is a novel antihepatitis drug developed by Chinese scientists (Figure 1) [6]. Clinical trials found that bicyclol is effective in improving abnormal liver function and in inhibiting the replication of $\mathrm{HBV}$ in chronic hepatitis B patients [7]. Pharmacologically, bicyclol exhibits a protective action against liver injury induced by hepatotoxins in mice and rats, an antifibrotic effect on $\mathrm{CCl}_{4}$ induced liver fibrosis in rats and mice, and an antihepatitis virus action in duck viral hepatitis and the 2.2 .15 cell line $[6,8,9]$. Furthermore, bicyclol induced differentiation of human hepatocarcinoma cells ( $\mathrm{HepG}_{2}$ and Bel-7402 cells) and reduced AFB1 hepatotoxicity in rats by increasing the detoxifying metabolism of AFB1 in rat liver [10]. In the latest study, bicyclol showed a significant inhibitory effect on the malignant transformation of WB-F344 rat liver epithelial cells induced by 3 -methylcholanthrene (3MC) and $12-O-$ tetradecanoylphorbol-13-acetate (TPA) [10]. These results implicate the possibility that bicyclol has a chemopreventive effect on liver carcinogenesis.

The present study aimed to further examine the effect of bicyclol on hepatocarcinogenesis induced by the chemical carcinogens diethylnitrosamine (DEN) as the carcinogenesis initiator and phenobarbital (PB) as the carcinogenesis promoter in mice.

\section{Materials and Methods}

2.1. Chemicals and Drugs. Bicyclol, a white crystalline powder with $99 \%$ purity, was kindly provided by the Beijing Union Pharmaceutical Plant and was suspended in 0.5\% sodium carboxymethylcellulose $(\mathrm{Na} \mathrm{CMC})$ for in vivo use. DEN, PB, dithiothreitol (DTT), phenylmethanesulfonyl fluoride (PMSF), aprotinin, leupeptin, $N, N^{\prime}$-methylene-bisacrylamide, and acrylamide were purchased from Sigma Chemical Co. (St. Louis, MO, USA). The kits for alanine aminotransferase (ALT), aspartate aminotransferase (AST), and alkaline phosphatase (ALP) determinations were purchased from Beihuakangtai Chemical Reagent Co., Ltd (Beijing, China). The ELISA kit for $\alpha$-fetal protein (AFP) determinations was offered by Shanghai Shenxiong Biotech
Company (Shanghai, China). All the other chemicals used were of analytical grade and were supplied by the Beijing Chemical Agents Company (Beijing, China).

2.2. Animals. Male ICR mice weighing $25-27 \mathrm{~g}$ ( 5 weeks old) were purchased from the Beijing Weitonglihua Experimental Animal Co., Ltd (Beijing, China). All mice were bred and maintained under constant conditions at a temperature of $24^{\circ} \mathrm{C} \pm 1{ }^{\circ} \mathrm{C}$ and humidity of $55 \% \pm 5 \%$ with $12 \mathrm{~h}$ light and $12 \mathrm{~h}$ dark cycles. Water and feed were accessible to the mice ad libitum. Animal care and all experimental procedures were conducted in accordance with the health criteria for care of laboratory animals enacted by the Beijing municipal government.

2.3. Experimental Protocol. The experimental mice were randomly divided into 4 groups, and each group contained 15 animals housed at 5 per cage. Liver cancers were induced in all the groups with a single i.p. injection of DEN at a dose of $100 \mathrm{mg} / \mathrm{kg}$ body weight in normal saline, except in the control group. After a treatment-free interval of 1 week, the mice were kept on $0.05 \%$ PB-containing water whereas the control group was kept on PB-free water for 19 weeks. To examine the prophylactic activities of bicyclol, the animals were pretreated with bicyclol $(100,200 \mathrm{mg} / \mathrm{kg})$ administered orally 2 days before DEN injection. After DEN injection, bicyclol was administered once daily for 6 days/week for a period of 20 weeks.

The mice were weighed periodically and their body weights were recorded. At week 20, 8-10 surviving mice of each group were sacrificed and 5 mice of each group were kept on fresh water and feed for another 10 weeks. Serum was obtained from the blood by centrifugation at $3000 \mathrm{rpm}$ for $10 \mathrm{~min}$. Liver specimens were removed carefully after the mice were sacrificed. Macroscopically visible liver tumors and nodules greater than approximately $1 \mathrm{~mm}$ in diameter on the liver surface were recorded. The right lobe of each liver and the nodules were fixed in paraformaldehyde solution for histopathological and immunohistochemical examinations. The remaining liver tissues were stored at $-80^{\circ} \mathrm{C}$ for performing biochemical, ELISA, and Western blot assays.

2.4. Histopathology. The fixed liver samples were processed and embedded in paraffin blocks. Tissue block sections were mounted on slides, deparaffinized in xylene, dehydrated in alcohol, and sections with a thickness of $5 \mu \mathrm{m}$ were prepared. Then, the sections were stained with hematoxylin and eosin (H\&E). Liver pathological changes and the extent of liver tumors were observed and diagnosed under a microscope by a histopathologist.

2.5. Biochemical Assays of AST, ALT, and ALP. The serum levels of AST, ALT, and ALP were measured using commercial kits (Beihuakangtai Chemical Reagent Co., Ltd, Beijing, China).

2.6. Enzyme-Linked Immunosorbent Assay (ELISA) of AFP. Quantitative estimation of the tumor marker AFP in liver 
and serum followed the corresponding protocols of the kits (Shanghai Shenxiong Biotech Company, Shanghai, China). Serum was obtained from blood by centrifugation at $3000 \mathrm{rpm}$ for $10 \mathrm{~min}$. Liver tissue was homogenized in an electron/grass homogenizer (DY89-I, Ningbo., China) using cold normal saline at $4^{\circ} \mathrm{C}$. Then, the homogenates were centrifuged. Aliquots of the supernatants and the serum were used to quantify AFP. The total proteins in liver homogenates were determined by the Lowry methods using bovine serum albumin as the standard. Optical density was measured using scanning full wavelength spectrophotometer (MQX200, BIO-TEK, USA).

2.7. Western Blot Analysis of PCNA. The liver tissue was homogenized in an electron/grass homogenizer (Dounce, Ningbo, China) using sodium dodecyl sulfate polyacrylamide gel electrophoresis (SDS-PAGE) lysis buffer $(50 \mathrm{mM}$ Tris-HCL, $150 \mathrm{mM} \mathrm{NaCl}, 5 \mathrm{mM}$ EDTA, 0.3\% Triton X-100, $0.03 \%$ SDS, $1 \mu \mathrm{g} / \mathrm{mL}$ aprotinin, $1 \mu \mathrm{g} / \mathrm{mL}$ leupeptin, $1 \mathrm{mM}$ PMSF) at $4^{\circ} \mathrm{C}$. After incubation on ice for $30 \mathrm{~min}$, the homogenates were centrifuged at $12000 \mathrm{~g}$ for $20 \mathrm{~min}$ and the supernatants were boiled in an SDS sample loading buffer for $4 \mathrm{~min}$ before electrophoresis on SDS-polyacrylamide gel. After electrophoresis for $2 \sim 3 \mathrm{~h}$, proteins in the SDSPAGE gel were transferred to nitrocellulose membranes at $27 \mathrm{~mA}$ for $12 \mathrm{~h}$ at $4^{\circ} \mathrm{C}$. The membranes were blocked in $5 \%$ milk-Tris buffered saline with Tween 20 (TBST) $(20 \mathrm{mM}$ Tris-HCl, pH 7.5, $500 \mathrm{Mm} \mathrm{NaCl}, 0.1 \%$ Tween 20 ) at $4{ }^{\circ} \mathrm{C}$ overnight. The blot was probed with a mouse anti-PCNA monoclonal antibody (Santa Cruz Biotechnology Inc., USA) at a dilution of $1: 200$ in 5\% milk-TBST for $2 \mathrm{~h}$ at room temperature and then incubated with an alkaline-phosphataseconjugated secondary antibody in skim milk-TBST for $1.5 \mathrm{~h}$ at room temperature. The blot was developed using the 5bromo-4-chloro-3-indolyl phosphate-nitro blue tetrazolium (BCIP/NBT) colorimetric method, and the densities of the bands were determined using the Gel-Pro Analyzer 4.0 software.

2.8. Immunohistochemistry. The paraffin-embedded liver tissue was cut into $5 \mu \mathrm{m}$ thick sections, deparaffinized, and incubated in $3 \% \mathrm{H}_{2} \mathrm{O}_{2}$ for $10 \mathrm{~min}$ to quench endogenous peroxidase activity. After blocking with normal goat serum for $20 \mathrm{~min}$, the sections were stained with a mouse antiAFP monoclonal antibody (Sigma Chemical Co., USA) and mouse anti-PCNA monoclonal antibody (Santa Cruz Biotechnology Inc., USA) at $4^{\circ} \mathrm{C}$ overnight, followed by incubation with a horseradish-peroxidase-conjugated goat anti-mouse antibody at $37^{\circ} \mathrm{C}$ for $30 \mathrm{~min}$. The antibody binding sites were visualized by incubation with diaminobenzidine (DAB) $-\mathrm{H}_{2} \mathrm{O}_{2}$ at room temperature for $10 \mathrm{~min}$. Digital images of AFP and PCNA immunoreactive liver cells were acquired at 100x magnification on an Olympus microscope (Olympus, Tokyo, Japan) using an attached Polaroid digital microscope camera (Polaroid, Cambridge, MA, USA) and IBM PC.
2.9. Statistical Analysis. The data were presented as the mean \pm SD. ANOVA was used for multiple comparisons of groups. The statistical significance between groups was assessed by the paired Student's $t$-test. A value of $P<0.05$ was considered to be significant.

\section{Results}

The chemopreventive effect of bicyclol on DEN/PB-induced hepatocellular cancer was elucidated in male ICR mice. At week 20, DEN/PB-induced tumors or nodules could be readily observed by unaided eyes (Figure 2(a)). All macroscopically visible liver nodules greater than approximately $1 \mathrm{~mm}$ in diameter were counted. Oral administration of bicyclol before tumor initiation resulted in a marked inhibition of tumor development in the animals. The average number of nodules per liver in the bicyclol-treated groups was significantly lower than those in the DEN/PB model group (Figure 2(b)). In addition, the body weight of the animals decreased on DEN/PB administration when compared to the control group. Pretreatment with bicyclol prevented the decrease in body weight (Figure 3).

In the histopathological examination of liver tissue at week 20, the control group animals revealed normal architecture. DEN/PB-induced animals showed loss of architecture and presence of tumor cells. Animals pretreated with bicyclol $(100,200 \mathrm{mg} / \mathrm{kg})$ showed fewer neoplastic cells, near-normal architecture, and significant improvement in liver histopathology (Figure 4). The tumor extent was diagnosed and evaluated by a histopathologist. As shown in Table 1, DEN initiation followed by PB promotion resulted in 33.3\% hepatocellular cancer, $55.6 \%$ hepatoma, and $88.9 \%$ multiple bile canaliculi hyperplasia formations in the liver. Preadministration of bicyclol showed a significant reduction in the incidence of liver tumors. Bicyclol administered at a dose of $200 \mathrm{mg} / \mathrm{kg}$ exhibited a stronger preventive effect on liver carcinogenesis as hepatocellular cancer and hepatoma were not observed in the mice at week 20 , but $50.0 \%$ lowgrade multiple bile canaliculi hyperplasia formations were observed.

Table 2 shows the levels of serum marker enzymes: ALT, AST, and ALP. DEN initiation and PB promotion resulted in a marked increase in serum ALT and ALP levels as compared to those in the control group whereas no marked changes were observed in the serum AST levels. Pretreatment with $100 \mathrm{mg} / \mathrm{kg}$ bicyclol reduced serum ALT levels significantly. However, bicyclol administered at a dose of $200 \mathrm{mg} / \mathrm{kg}$ failed to considerably change the abovementioned levels of ALT, AST, and ALP.

Figure 5(b) depicts the levels of the tumor marker AFP in serum and liver by using the ELISA assay. DEN initiation followed by $\mathrm{PB}$ promotion resulted in a marked increase in AFP levels in liver and serum as compared with that of the control group. Pretreatment with bicyclol significantly decreased the AFP level. Bicyclol also reduced the serum AFP level, while there was no statistical significance when compared with the model group because of the large standard deviation. In the immunohistochemistry assay (Figure 5(a)), the AFP 


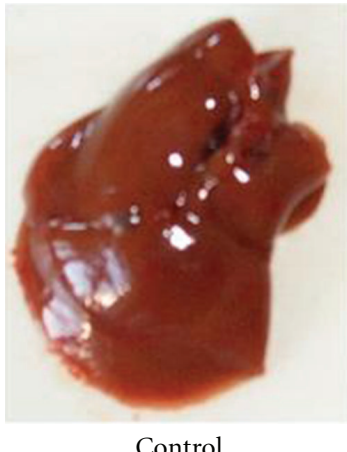

Control

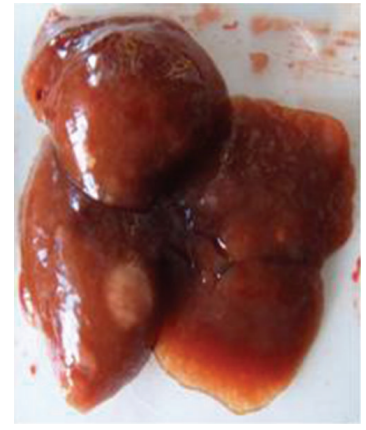

DEN/PB model

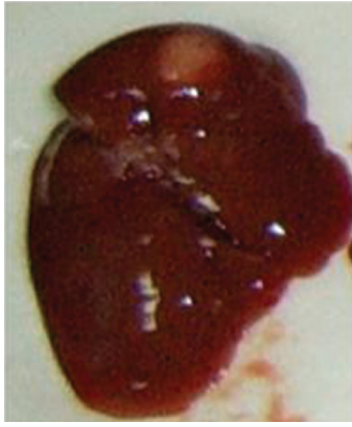

Bicyclol $100 \mathrm{mg} / \mathrm{kg}$ $+\mathrm{DEN} / \mathrm{PB}$

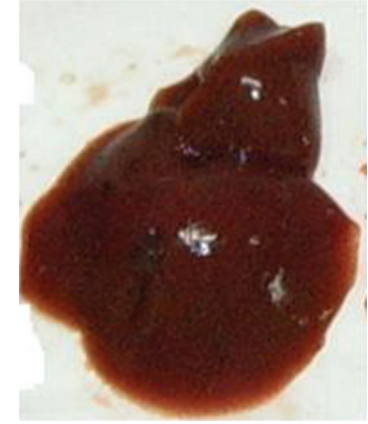

Bicyclol $200 \mathrm{mg} / \mathrm{kg}$ $+\mathrm{DEN} / \mathrm{PB}$

(a)



(b)

Figure 2: Gross liver tumor and nodule phenotypes in DEN/PB-induced ICR mice and the preventive effect of bicyclol at week 20. (a) Photographs of representative livers. (b) Determination of liver tumor and number of nodules per liver greater than approximately 1 mm in diameter. Average values with standard deviations are shown. $n=8-10$. ${ }^{\#} P<0.01$ compared with the control group; ${ }^{*} P<0.01,{ }^{* *} P<0.01$ compared with the DEN/PB model group.

TABLE 1: The incidence of liver tumors induced by DEN/PB in mice and the effect of bicyclol determined by H\&E staining and histological evaluation.

\begin{tabular}{|c|c|c|c|c|c|c|}
\hline Group & $\begin{array}{l}\text { Dosage } \\
(\mathrm{mg} / \mathrm{kg})\end{array}$ & $\mathrm{HCC}$ & Hepatoma & Cholangioadenoma & $\begin{array}{c}\text { Capillary } \\
\text { hemangioma }\end{array}$ & MBCA \\
\hline Control & - & $0.0(0 / 6)$ & $0.0(0 / 6)$ & $0.0(0 / 6)$ & $0.0(0 / 6)$ & $0.0(0 / 6)$ \\
\hline $\mathrm{DEN} / \mathrm{PB}$ & - & $33.3(3 / 9)$ & $55.6(5 / 9)$ & $22.2(2 / 9)$ & $11.1(1 / 9)$ & $88.9(8 / 9)$ \\
\hline \multirow[t]{2}{*}{ Bicyclol } & 100 & $11.1(1 / 9)$ & $22.2(2 / 9)$ & $11.1(1 / 9)$ & $0.0(0 / 9)$ & $33.3(3 / 9)$ \\
\hline & 200 & $0.0(0 / 8)$ & $0.0(0 / 8)$ & $0.0(0 / 8)$ & $0.0(0 / 8)$ & $50.0(4 / 8)$ \\
\hline
\end{tabular}

HCC: hepatocellular carcinoma; MBCA: multiple bile canaliculi hyperplasia.

immunoreactivity in Page: 9 DEN/PB-induced liver cells was more intense than that in the control liver cells. Pretreatment with bicyclol markedly decreased the expression of AFP. Using the same ELISA kit, we also found that bicyclol did not exhibit any lowering effect on the physiological elevation of serum AFP in pregnant mice and fetal mice livers treated with bicyclol $200 \mathrm{mg} / \mathrm{kg}$ for 10 days (Figure 5(c)).

Figure 6 reveals the immunohistochemical and Western blot analysis of PCNA. In immunohistochemical analysis (Figure 6(a)), the liver sections of the control mice stained for PCNA showed few nuclear-positive cells. However, DEN/PB treatment remarkably increased not only the intensity of immunostaining but also the number of PCNA-positive hepatocytes, which were both significantly reduced with bicyclol pretreatment. In the Western blot assay, preadministered bicyclol also decreased the increased expression of PCNA induced by DEN/PB (Figure 6(b)).

In this study, we also found that 10 weeks after stopping the administration of PB and drugs, the control and bicycloltreated $(200 \mathrm{mg} / \mathrm{kg})$ animals showed no HCC and hepatoma formation at the time of termination whereas DEN/PBinduced mice developed 100\% hepatoma and 50\% HCC (data not shown).

\section{Discussion}

DEN is a potent hepatocarcinogenic nitrosamine present in tobacco smoke, water, cheddar cheese, cured and fried meats, 
TABLE 2: Levels of serum marker enzymes ALT, AST, and ALP in all groups.

\begin{tabular}{lcccc}
\hline Group & $\begin{array}{c}\text { Dosage } \\
(\mathrm{mg} / \mathrm{kg})\end{array}$ & AST $(\mathrm{u} / \mathrm{L})$ & ALT $(\mathrm{u} / \mathrm{L})$ & ALP $(\mathrm{u} / \mathrm{L})$ \\
\hline Control & - & $332.0 \pm 32.8$ & $182.9 \pm 25.7$ & $5.3 \pm 1.2$ \\
DEN/PB & - & $334.1 \pm 32.1$ & $283.5 \pm 55.9^{\# \#}$ & $10.8 \pm 6.4^{\#}$ \\
Bicyclol & 100 & $324.3 \pm 19.1$ & $224.4 \pm 23.3^{* *}$ & $8.4 \pm 4.3$ \\
& 200 & $350.5 \pm 45.6$ & $222.2 \pm 53.8$ & $7.8 \pm 2.2$ \\
\hline
\end{tabular}

Data are means \pm SD values $(n=8 \sim 10) .{ }^{\#} P<0.05,{ }^{\# \#} P<0.01$, compared with the control group. ${ }^{* *} P<0.01$ compared with the DEN/PB model group.

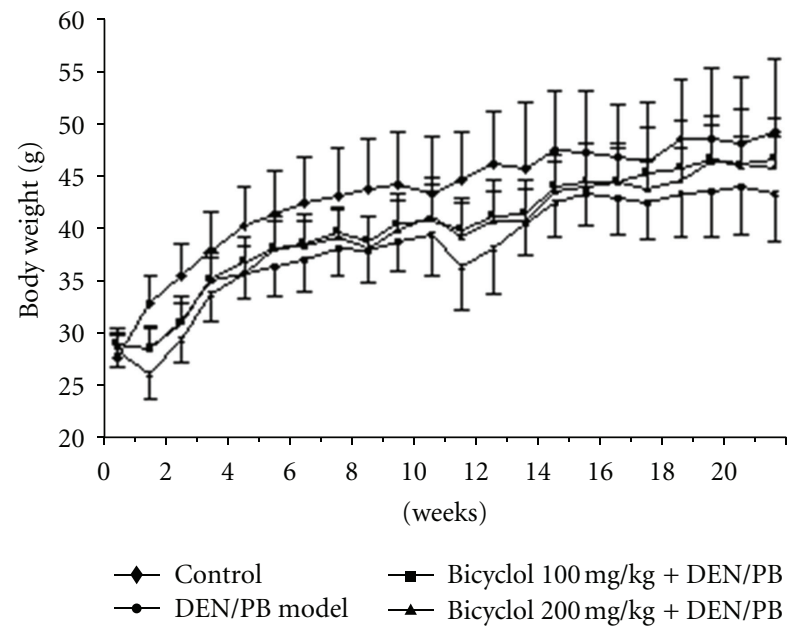

Figure 3: Body weight profile in the control and experimental groups. Average values with standard deviations are shown. $n=14-$ 15.

occupational settings, cosmetics, agricultural chemicals, and pharmaceutical agents. DEN-induced HCC is one of the most accepted and widely used experimental models to study hepatocarcinogenesis [11]. There are 2 liver tumorigenesis protocols for DEN. DEN treatment at the neonatal period (2-week-old mice administered with DEN at a dose of $5 \mathrm{mg} / \mathrm{kg}$ body weight) causes severe hepatocyte toxicity. DEN treatment of young adult mice (4-5-week-old mice administered with DEN at a dose of $100 \mathrm{mg} / \mathrm{kg}$ body weight) is less toxic and requires tumor promotion by long-term treatment with $\mathrm{PB}$, a procedure referred to as the two-stage protocol [12-14].

In this study, we used the DEN/PB two-stage protocol to determine whether bicyclol has a chemopreventive effect on hepatocarcinogenesis in vivo. As female mice are known to be resistant to hepatocarcinogenesis in experimental mouse models, including those employing chemical carcinogenesis, only male mice were used and analyzed in our experiments [15]. In this study, we found that pretreatment with bicyclol $(100,200 \mathrm{mg} / \mathrm{kg}$ orally) markedly decreased the number of liver tumor nodules per liver. According to the histopathological diagnosis, the model control group developed 33.3\% HCC and 55.6\% hepatoma at week 20. However, the mice pretreated with $200 \mathrm{mg} / \mathrm{kg}$ bicyclol showed no HCC and hepatoma formation in their liver. Ten weeks after stopping administration of the promoter and bicyclol, there was still no tumor formation in the $200 \mathrm{mg} / \mathrm{kg}$ bicyclol group whereas $100 \%$ hepatoma and 50\% HCC was observed in the DEN/PB model group. These results further revealed that bicyclol had a preventive effect on hepatocarcinogenesis induced by carcinogens and this preventive activity is persistent.

Liver damage caused by DEN and PB could result in the deterioration or destruction of the cell membrane which leads to the leakage of transaminases and ALP from the liver tissue. The serum levels of transaminases and ALP are representative of the liver function; their increased levels are indicators of liver damage. Cancer chemoprevention and therapy depends on the investigation of these marker enzymes [16]. The elevation of ALT levels is repeatedly credited to hepatocellular damage and is usually accompanied by a rise in AST levels. Increase in ALP levels reflects the pathological alteration in biliary flow. In the present study, pretreatment with bicyclol attenuated the increased activities of ALT and ALP induced by DEN/PB, but only bicyclol$(100 \mathrm{mg} / \mathrm{kg})$ treated mice showed statistically significant results when compared with the DEN/PB group. There were no changes in serum AST levels in the model and bicycloltreated groups. It is shown that bicyclol helps regenerate the parenchymal cells in liver, protecting membrane integrity and thereby decreasing enzyme leakage (results in [6]). These results shown in this paper also suggest that the protective effect on cell membranes is not the major mechanism by which the chemopreventive effect of bicyclol is exerted.

AFP, an oncofetal serum protein, is progressively lost during development, such that it is virtually absent from a healthy adult $[17,18]$. It has been widely used as a clinical marker in the diagnosis and monitoring of HCC. As we previously reported in human hepatocellular carcinoma MHCC97-H cells with high metastatic potential [19], bicyclol pretreatment significantly decreased the production and secretion of AFP. Since AFP overexpression has been associated with uncontrolled growth of HCC [20], our data suggests that bicyclol prevented the progression of HCC that is associated with the downregulation of cell proliferation. Another finding in this study is that bicyclol does not significantly affect the physiological AFP levels in pregnant mice and fetal mice livers. These findings indicate that the lowering effect of bicyclol on AFP is selective. This is an interesting finding, and further studies need to be carried out. 

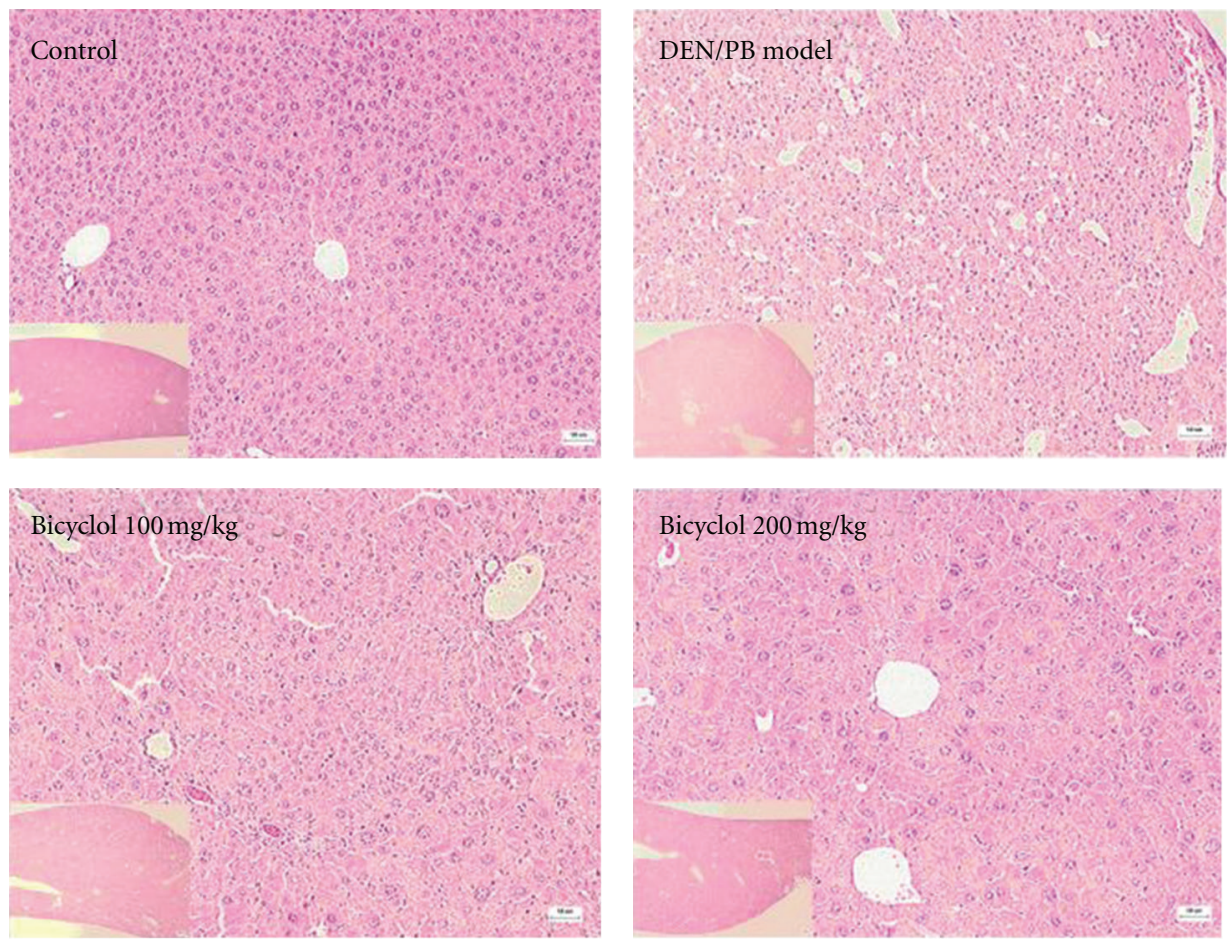

FIGURE 4: Histological study of the liver tissue obtained from the control and experimental groups at week 20. H\&E-stained liver sections were photographed at 100x magnification. Insets in the bottom-left corners are images at 20x magnification of the liver tissue from which the 100x photographs were taken. Control group animals revealed normal architecture. DEN/PB-induced animals showed loss of architecture and presence of cancer cells. Animals pretreated with bicyclol (100, $200 \mathrm{mg} / \mathrm{kg})$ showed fewer neoplastic cells and near-normal architecture.

PCNA is another marker of cell proliferation. It plays an essential role in nucleic acid metabolism as a component of the replication and repair machinery [21]. This toroidal-shaped protein encircles the DNA and can slide bidirectionally along the duplex. PCNA has been identified as the DNA polymerase delta accessory protein. In our studies, the expression of PCNA significantly increased in DEN/PB-induced mice in terms of the number of PCNApositive hepatocytes examined by immunohistochemistry, and the expression of PCNA was quantified by Western blot assay. Pretreatment with bicyclol decreased the expression of PCNA. This data suggests that bicyclol prevents the development of liver tumors along with the inhibition of cell proliferation.

In addition, cytochrome P450 (CYP) monooxygenase also plays a critical role in the activation of various chemical toxicants and precarcinogens [22-24]. Metabolic activation of DEN by CYP enzymes to form reactive electrophiles is required for its toxicity, and $\mathrm{PB}$ induces a large spectrum of drug-metabolizing enzymes [25]. According to the results of previous studies [26-28], bicyclol had the modulating activity on CYP isozymes, including the mild inhibition to CYP2C and CYP2D, the mild induction to CYP2E1, and reducing the metabolic rates of the selective substrates of CYP3A, $1 \mathrm{~A} 2,2 \mathrm{E} 1$, and 2C. Bicyclol could enhance particularly the denitrosation of DEN, a low toxic pathway of metabolism. Based on these preliminary results, the modulating activity on CPY isozymes of bicyclol may also partly contribute to the prevention efficacy on hepatocarcinogenesis induced by $\mathrm{DEN}$ and $\mathrm{PB}$ found in this paper.

\section{Conclusions}

In summary, the findings of the present in vivo study are consistent with the results of our in vitro studies where we found that bicyclol prevents the malignant transformation of WB-F344 cells induced by 3MC and TPA [10]. Taken together, bicyclol has chemopreventive potential to the liver carcinogenesis induced by carcinogens. This is an interesting finding, and further studies on whether bicyclol can prevent liver carcinogenesis induced by chronic viral hepatitis need to be carried out. Additional studies to understand other molecular mechanisms responsible for its chemopreventive efficacy are warranted to claim bicyclol as a potential chemopreventive agent against liver cancer induced by carcinogens.
Abbreviations
AFP: $\quad \alpha$-fetal protein
ALP: Alkaline phosphatase
ALT: Alanine aminotransferase
AST: Aspartate aminotransferase
ELISA: Enzyme-linked immunosorbent assay
DEN: Diethylnitrosamine
HBV: Hepatitis B virus
HCC: Hepatocellular carcinoma 

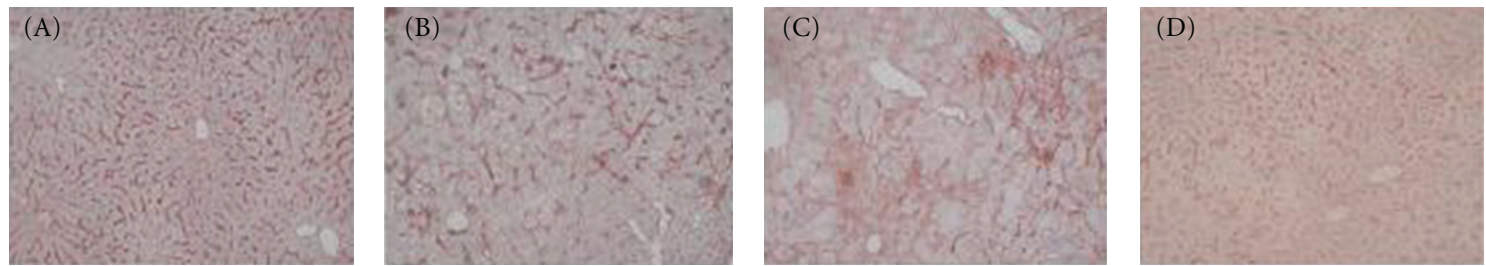

(a)
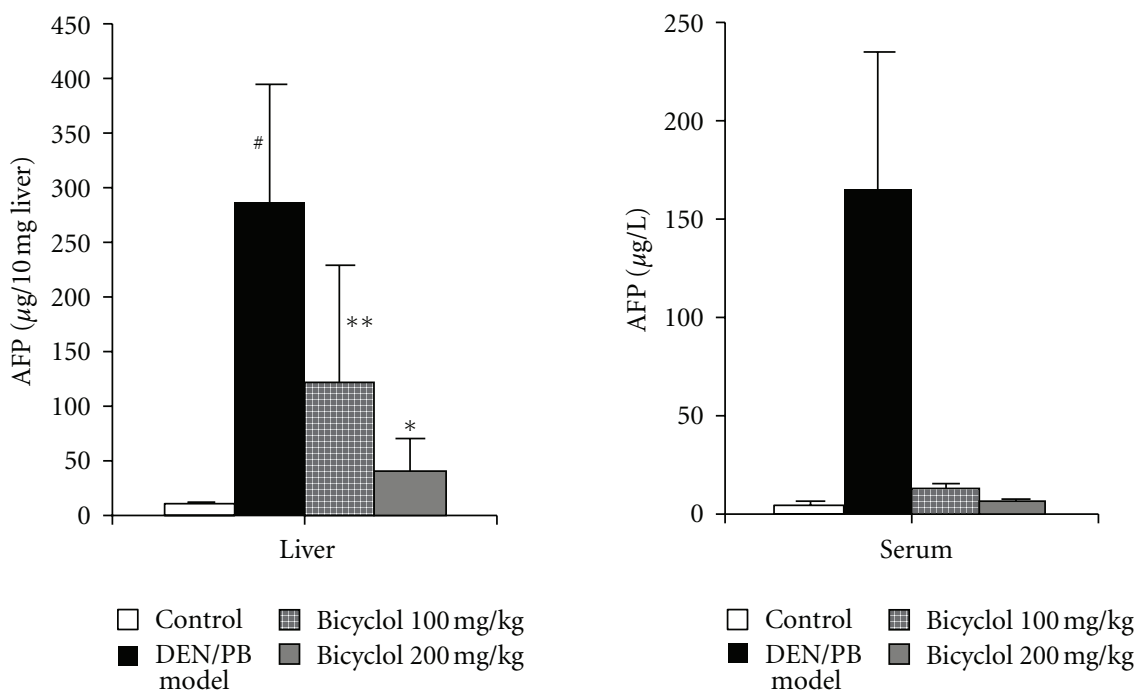

(b)
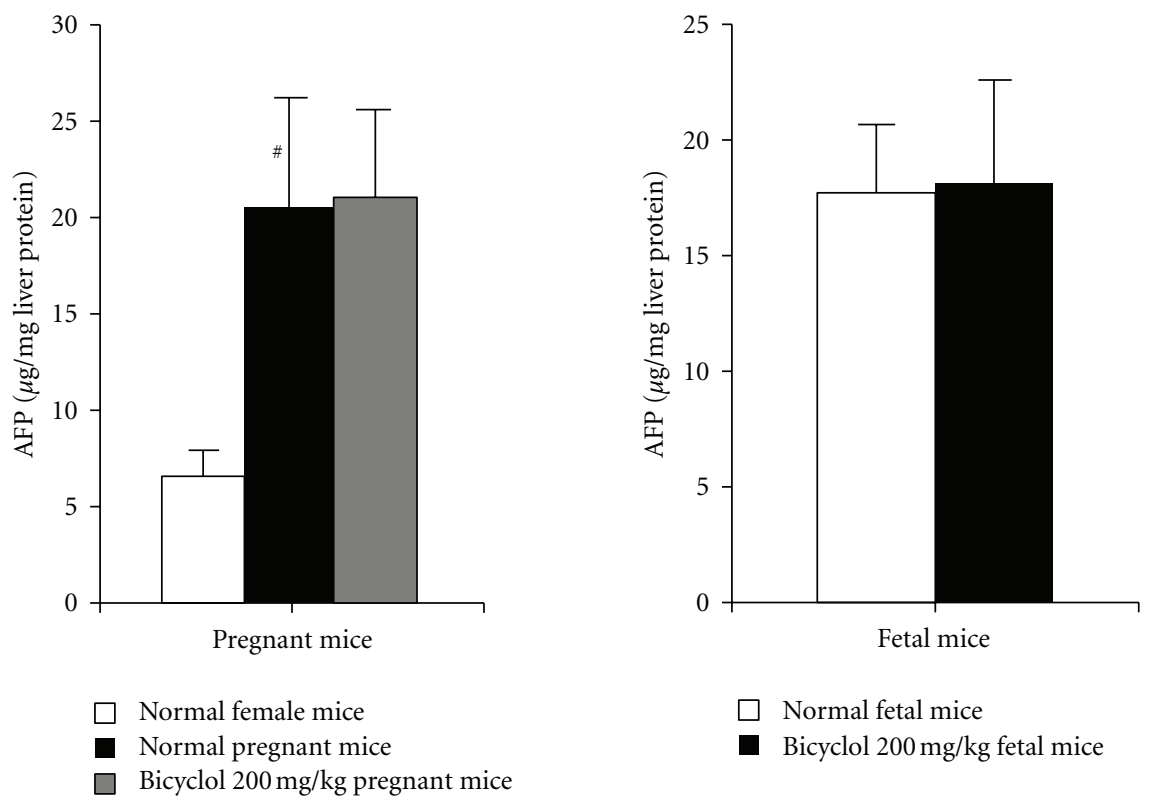

(c)

FigURE 5: Expression and quantification of $\alpha$-fetal protein (AFP). (a) AFP-immunoreactivity assay in the control and experimental groups at week 20 using immunohistochemistry assay. (A) control; (B) DEN/PB model; (C) treatment with bicyclol (100 mg/kg) before DEN injection; (D) treatment with bicyclol (200 mg/kg) before DEN injection. (b) Quantification of serum and tissue AFP levels in the control and experimental groups at week 20 using an enzyme immunoassay kit. Average values with standard deviations are shown. $n=8-10$. ${ }^{\#} P<0.05$ compared with the control group; ${ }^{*} P<0.05,{ }^{*} P<0.01$ compared with the DEN/PB model group. (c) Quantification of liver AFP levels in pregnant mice and fetal mice using an enzyme immunoassay kit. Average values with standard deviations are shown. $n=4$. ${ }^{\#} P<0.05$ compared with the control group. 

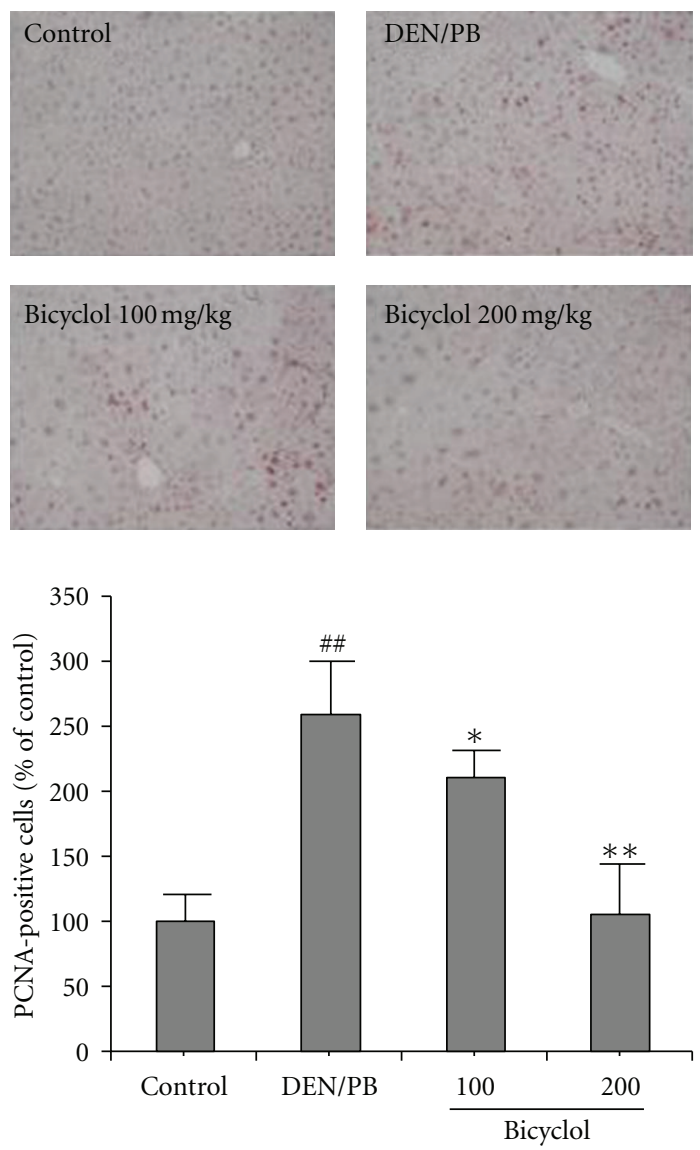

(a)
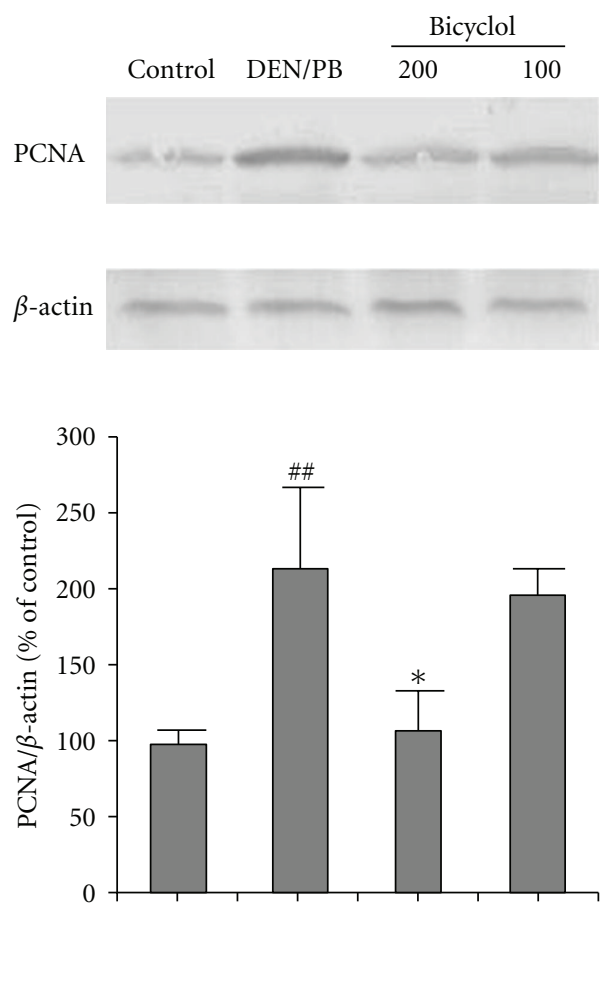

(b)

Figure 6: Expression of PCNA in the control and experimental groups at week 20. (a) Representative microscopic pictures of PCNA-stained liver sections by immunohistochemical analysis $(100 \times)$ and quantification of the immunostained section of PCNA. \# $P<0.01$ compared with the control group; ${ }^{*} P<0.05$, ${ }^{* *} P<0.01$ compared with the DEN/PB model group. (b) Western blot assay of PCNA and the quantitative results obtained by measuring the optical density of each band and expressed as the ratio of each targeted protein and beta-actin. Average values of 3 separate experiments along with the standard deviations are shown. ${ }^{\# \#} P<0.01$ compared with the control group; ${ }^{*} P<0.05$ compared with the DEN/PB model group.

HCV: Hepatitis C virus

3MC: 3-Methylcholanthrene

PB: Phenobarbital

TPA: 12-O-tetradecanoylphorbol-13-acetate.

\section{Acknowledgments}

The authors would like to dedicate this paper to the memory of Academician Geng Tao Liu, who unfortunately passed away during the preparation of this paper. They thank Professor Yongrong Zhang for performing histological diagnoses. This work was supported by Grants (no. 30801409) from China National Natural Sciences Foundation.

\section{References}

[1] M. A. Feitelson, J. Pan, and Z. Lian, "Early molecular and genetic determinants of primary liver malignancy," Surgical Clinics of North America, vol. 84, no. 2, pp. 339-354, 2004.
[2] L. M. Yerian, R. A. Anders, M. Tretiakova, and J. Hart, "Caveolin and thrombospondin expression during hepatocellular carcinogenesis," American Journal of Surgical Pathology, vol. 28, no. 3, pp. 357-364, 2004.

[3] R. B. Birrer, D. Birrer, and J. V. Klavins, "Review: hepatocellular carcinoma and hepatitis virus," Annals of Clinical and Laboratory Science, vol. 33, no. 1, pp. 39-54, 2003.

[4] R. S. Brown and P. J. Gaglio, "Scope of worldwide hepatitis C problem," Liver Transplantation, vol. 9, no. 11, pp. S10-S13, 2003.

[5] B. Glinghammar, J. Shogsberg, A. Hamsten, and E. Ehrenborg, "PPAR $\delta$ activation induces COX-2 gene expression and cell proliferation in human hepatocellular carcinoma cells," Biochemical and Biophysical Research Communications, vol. 308, pp. 361-368, 2003.

[6] Y. Li, G. W. Dai, Y. Li, and G. T. Liu, "Effect of bicyclol on acetaminophen-induced hepatotoxicity: energetic metabolism and mitochondrial injury in acetaminophenintoxicated mice," Yaoxue Xuebao, vol. 36, no. 10, pp. 723-726, 2001 (Chinese). 
[7] G. T. Liu, C. Z. Zhang, Y. Li, H. L. Wei, and W. Hu, "Clinical and pharmacological study of bicyclol: a potential antichronic viral hepatitis drug," Chinese Medical Sciences Journal, vol. 14, pp. 51-53, 1999 (Chinese).

[8] M. Li and G. T. Liu, "Inhibition of Fas/FasL mRNA expression and TNF- $\alpha$ release in concanavalin A-induced liver injury in mice by bicyclol," World Journal of Gastroenterology, vol. 10, no. 12, pp. 1775-1779, 2004.

[9] D. Zhao and G. Liu, "Protective effect of bicyclol on concanavalin A-induced liver nuclear DNA injury in mice," Zhong Hua Yi Xue Za Zhi, vol. 81, no. 14, pp. 844-848, 2001.

[10] H. Sun and G. T. Liu, "Chemopreventive effect of bicyclol on malignant transformation of WB-F344 rat liver epithelial cells and its effect on related signal transduction in vitro," Cancer Letters, vol. 236, no. 2, pp. 239-249, 2006.

[11] V. Sivaramakrishnan, P. N. M. Shilpa, V. R. Praveen Kumar, and S. Niranjali Devaraj, "Attenuation of N-nitrosodiethylamine-induced hepatocellular carcinogenesis by a novel flavonol-Morin," Chemico-Biological Interactions, vol. 171, no. 1, pp. 79-88, 2008.

[12] D. S. Sarma, P. M. Rao, and S. Rajalakshmi, "Liver tumour promotion by chemicals: models and mechanisms," Cancer Surveys, vol. 5, no. 4, pp. 781-798, 1986.

[13] D. Sun, H. Ren, M. Oertel, R. S. Sellers, D. A. Shafritz, and L. Zhu, "Inactivation of p27Kip1 promotes chemical mouse liver tumorigenesis in the resistant strain C57BL/6J," Molecular Carcinogenesis, vol. 47, no. 1, pp. 47-55, 2008.

[14] G. J. Kapadia, M. A. Azuine, R. Sridhar et al., "Chemoprevention of DMBA-induced UV-B promoted, NOR-1-induced TPA promoted skin carcinogenesis, and DEN-induced phenobarbital promoted liver tumors in mice by extract of beetroot," Pharmacological Research, vol. 47, no. 2, pp. 141-148, 2003.

[15] T. Nakatani, G. Roy, N. Fujimoto, T. Asahara, and A. Ito, "Sex hormone dependency of diethylnitrosamine-induced liver tumors in mice and chemoprevention by leuprorelin," Japanese Journal of Cancer Research, vol. 92, no. 3, pp. 249256, 2001.

[16] V. Sivaramakrishnan, P. N. M. Shilpa, V. R. Praveen Kumar, and S. Niranjali Devaraj, "Attenuation of Nnitrosodiethylamine-induced hepatocellular carcinogenesis by a novel flavonol-Morin," Chemico-Biological Interactions, vol. 171, no. 1, pp. 79-88, 2008.

[17] B. Daniele, A. Bencivenga, A. S. Megna, and V. Tinessa, " $\alpha$ fetoprotein and ultrasonography screening for hepatocellular carcinoma," Gastroenterology, vol. 127, pp. S108-S112, 2004.

[18] D. Bader, A. Riskin, O. Vafsi et al., "Alpha-fetoprotein in the early neonatal period-a large study and review of the literature," Clinica Chimica Acta, vol. 349, no. 1-2, pp. 15-23, 2004.

[19] H. Sun and G. T. Liu, "Inhibitory effect of anti-hepatitis drug bicyclol on invasion of human hepatocellular carcinoma MHCC97-H cells with high metastasis potential and its relative mechanisms," Journal of Asian Natural Products Research, vol. 11, no. 6, pp. 576-583, 2009.

[20] W. Cui, F. Gu, and K. Q. Hu, "Effects and mechanisms of silibinin on human hepatocellular carcinoma xenografts in nude mice," World Journal of Gastroenterology, vol. 15, no. 16, pp. 1943-1950, 2009.

[21] Z. Kelman, "PCNA: structure, functions and interactions," Oncogene, vol. 14, no. 6, pp. 629-640, 1997.

[22] X. Zhao, J. J. Zhang, X. Wang, X. Y. Bu, Y. Q. Lou, and G. L. Zhang, "Effect of berberine on hepatocyte proliferation, inducible nitric oxide synthase expression, cytochrome $\mathrm{P} 4502 \mathrm{E} 1$ and $1 \mathrm{~A} 2$ activities in diethylnitrosamine- and phenobarbital-treated rats," Biomedicine and Pharmacotherapy, vol. 62, no. 9, pp. 567-572, 2008.

[23] F. P. Guengerich, "Forging the links between metabolism and carcinogenesis," Mutation Research, vol. 488, no. 3, pp. 195209, 2001

[24] J. A. Hasler, R. Estabrook, M. Murray et al., "Human cytochromes P450," Molecular Aspects of Medicine, vol. 20, no. 1-2, pp. 1-137, 1999.

[25] H. L. Lin and P. F. Hollenberg, "N-nitrosodimethylaminemediated formation of oxidized and methylated DNA bases in a cytochrome P450 2E1 expressing cell line," Chemical Research in Toxicology, vol. 14, no. 5, pp. 562-566, 2001.

[26] B. Zhu, T. L. Geng, S. W. Ruo, and S. J. Strada, "Chemoprevention of bicyclol against hepatic preneoplastic lesions," Cancer Biology and Therapy, vol. 5, no. 12, pp. 1665-1673, 2006.

[27] X. M. Yao, B. L. Wang, Y. Gu, and Y. Li, "Effects of bicyclol on the activity and expression of CYP450 enzymes of rats after partial hepatectomy," Yao Xue Xue Bao, vol. 46, pp. 656-663, 2011.

[28] J. P. Hu, H. Chen, and Y. Li, "Effect of bicyclol on liver microsomal cytochrome P450 isozymes and phase II enzymes in rats," Chinese Journal of New Drugs, vol. 18, no. 4, pp. 340348, 2009. 


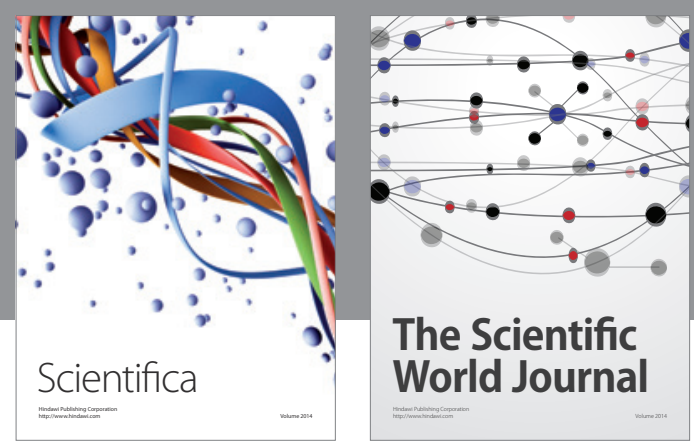

The Scientific World Journal
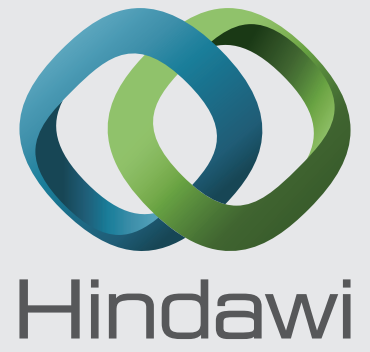

Submit your manuscripts at

http://www.hindawi.com
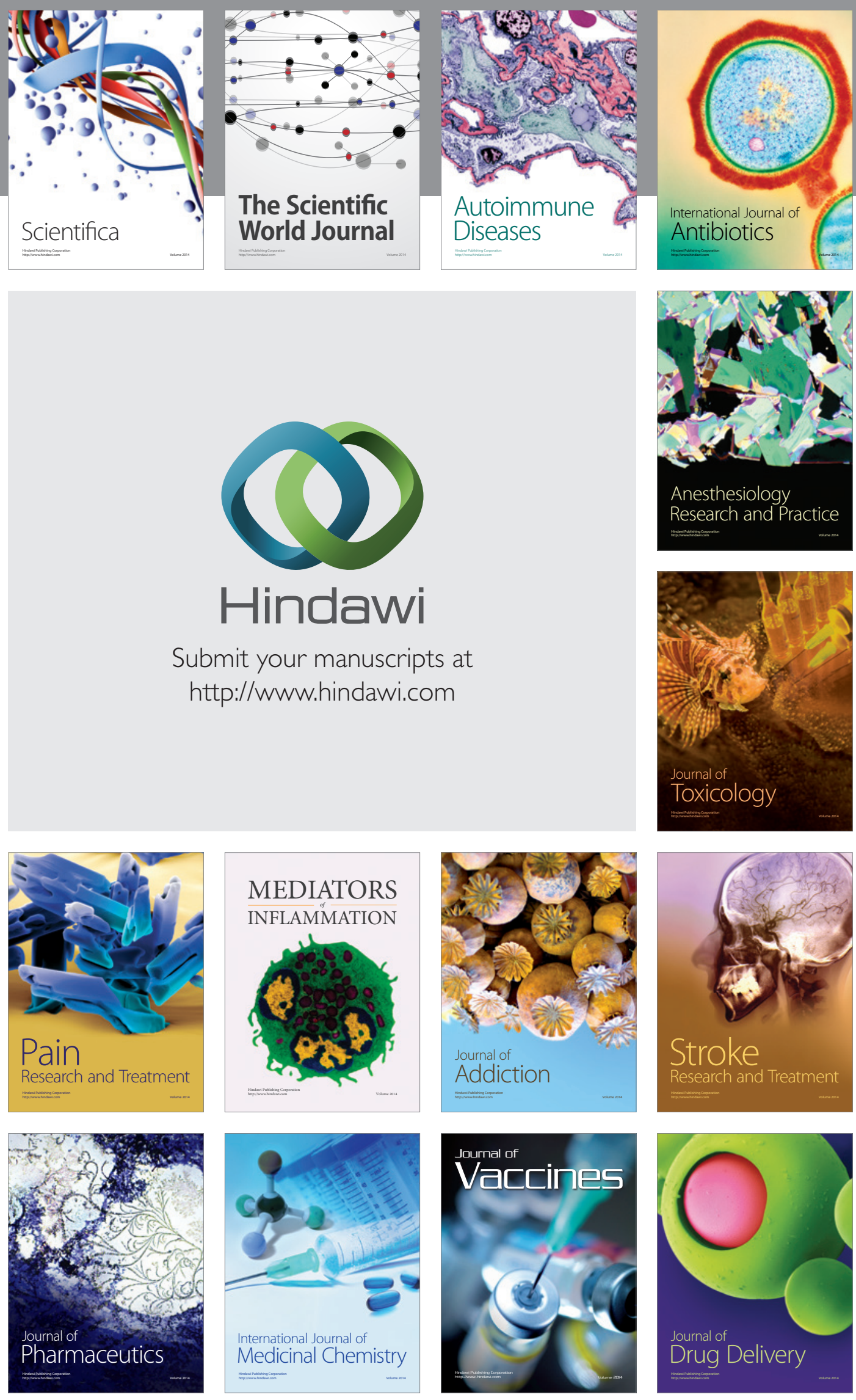